\title{
Presencia del alga Chlamydomomas vesterbottnica (Chlamydomonadales: Chlamydomonadaceae) en los estanques de la planta de tratamiento de un relleno sanitario en Costa Rica
}

\author{
Carola Scholz y Narcy Villalobos \\ Escuela de Ciencias Biológicas. Universidad Nacional, Heredia, Costa Rica; carola.scholz@una.cr
}

Recibido 26-XI-2012 Corregido 21-III-2013 Aceptado 26-IV-2013

\begin{abstract}
Ocurrence of the alga Chlamydomomas vesterbottnica (Chlamydomonadales: Chlamydomonadaceae) in the water treatment ponds of a landfill in Costa Rica. Algae belonging to the genus Chlamydomonas have a cosmopolitan distribution and many of them have the ability to adapt to extreme environmental conditions. These may even be found in ponds containing wastewater of domestic and industrial origin, which may have high nutrient levels as well as concentrations of organic and inorganic contaminants. In this work we report for the first time in Costa Rica the presence of a green algae species, Chlamydomonas vesterbottnica, located in ponds of a treatment plant receiving leachate from Los Mangos landfill in Alajuela. The presence of this alga arouses special interest for possible biotechnological applications with wastewater from treatment plants.
\end{abstract}

\section{KEY WORDS}

Microalgae, Chlamydomonas vesterbottnica Skuja, effluents, sanitary landfill

\section{RESUMEN}

Las algas que pertenecen al género Chlamydomonas tienen una distribución cosmopolita y muchas de ellas se reconocen por su capacidad de adaptarse a condiciones ambientales extremas. Estas inclusive se pueden encontrar en estanques que contienen aguas residuales de orígenes domésticos e industriales, los cuales pueden tener altos niveles de nutrientes y concentraciones de contaminantes orgánicos e inorgánicos. En este trabajo se reporta por primera vez en Costa Rica, la presencia de una especie de las algas verdes, Chlamydomonas vesterbottnica, encontrada en los estanques de la planta de tratamiento que recibe los lixiviados generados en el Relleno Sanitario de Los Mangos en Alajuela. La presencia de esta alga despierta un especial interés para buscar posibles aplicaciones biotecnológicas de aguas residuales en plantas de tratamientos.

\section{PALABRAS CLAVE}

Microalgas, Chlamydomonas vesterbottnica Skuja, aguas residuales, rellenos sanitarios
Las microalgas se pueden encontrar en todas partes, donde la luz y el agua son elementos disponibles. La biodiversidad de este grupo de organismos que en su mayoría son fotoautotróficos, es extraordinaria, y actualmente se reportan 128093 especies en el mundo (Guiry \& Guiry, 2011). Pero no sólo su riqueza y su distribución cosmopolita son sorprendentes, también destaca la capacidad de este grupo de seres vivos de adaptarse a condiciones ambientales extremas, ya que mediante sus mecanismos bioquímicos específicos, logran vivir en aguas saladas o salobres bajo los efectos de las fluctuaciones de salinidad, así como también sobre rocas, en el suelo, sobre el hielo del ártico, o en el desierto, adaptados a periodos de ausencia completa de agua (Graham, Wilcox \& Graham, 2008).

Esta capacidad de adaptación a condiciones ambientales extremas les ha permitido adaptarse fácilmente a zonas urbanas e industriales, especialmente aquellos espacios que reciben aguas residuales domésticas e industriales, y que presentan condiciones ambientales difíciles para la vida de muchos organismos, debido principalmente a los altos contenidos de contaminantes generados por el hombre.

Uno de estos sitios, en donde se generan aguas muy contaminadas, son los rellenos sanitarios que sirven para la deposición de los residuos sólidos de origen doméstico 
e industrial. Una vez enterrados estos desechos, se inician los procesos de su descomposición los cuales liberan sustancias que originalmente estaban dentro de los residuos. El agua que entra en contacto con la basura, recoge gran cantidad de estos componentes, quedando de esa manera altamente contaminada. Este líquido, denominado lixiviado, es uno de los más contaminados que se conocen, debido a su alta concentración de contaminantes orgánicos e inorgánicos, incluyendo ácidos húmicos, nitrógeno amoniacal, metales pesados, compuestos xenobióticos y sales inorgánicas, los cuales deben ser eliminados debido a su toxicidad o efecto desfavorable sobre el ambiente (Ehrig, 1992; Giraldo, 2001).

Particularmente las microalgas del género Chlamydomonas, pertenecen al grupo de las algas verdes (Filum Chlorophyta) y tienen una distribución cosmopolita. Además son unicelulares móviles que poseen de dos a cuatro flagelos. Muchas de estas especies abundan en cuerpos de aguas pequeños, con niveles altos o muy altos de nutrientes, tales como en lagunas de oxidación para el tratamiento de aguas residuales; sin embargo, se sabe poco sobre su ecología (John, Whitton \& Brook, 2002). Las algas de este género son hasta ahora las más estudiadas y utilizadas a nivel de laboratorio en la biología molecular, ya que son utilizadas para producir proteínas recombinantes, debido principalmente a que las tecnologías actuales cuentan con una serie de herramientas y metodologías que permiten su manipulación genética de manera rutinaria (Harris, 2001; Manuell \& Mayfield, 2006).

El estudio se realizó en el Relleno Sanitario Los Mangos que se encuentra en la parte noroeste del cantón central de la provincia de Alajuela, y está operado por la empresa WPP (Grupo WPP Continental de Costa Rica, S.A.). El Relleno Los Mangos recibe en promedio 60 toneladas de basura por día y genera entre 60 y $80 \mathrm{~m}^{3}$ de lixiviados diariamente.

Este relleno posee una planta de tratamiento de sus aguas residuales, la cual está constituida por tres etapas: una primera que está conformado por un tanque que recolecta los lixiviados y a la vez tienen la función del sedimentador, una segunda que incluye un proceso físico-químico de los lixiviados, y una tercera que comprende un proceso biológico que consiste en un tratamiento anaeróbico.

Para la colecta de la muestras se realizaron tres visitas al relleno sanitario en diferentes épocas del año 2010, comprendiendo el inicio de la época seca, el período de transición y la época lluviosa. Las muestras de lixiviados fueron colectadas en la segunda etapa del sistema de tratamiento a una profundidad de $10 \mathrm{~cm}$ en un punto cercano a la salida del tanque, sitio que fue seleccionado ya que debido al flujo interno, presentaba la mayor acumulación de microalgas. El muestreo se realizó con un recipiente cuyo contenido era de $1 \mathrm{~L}$ del cual se extrajo una submuestra de $100 \mathrm{~mL}$, estas submuestras fueron depositadas en una hielera para su debido traslado al Laboratorio de Botánica de la Escuela Ciencias Biológicas para su revisión y almacenamiento.

Las muestras frescas se estudiaron inmediatamente en un microscopio Nikon Eclipse Ni-U equipado con una cámara Nikon DS_13 para la captura de las imágenes. Para la determinación de la microalga se utilizó la literatura especializada (John et al., 2002; Nozaki, 2003; Menezes \& de Mattos Bicudo, 2008). Las muestras fueron tratadas con los procedimientos para la obtención de cultivos unialgales descritos en Anderson (2005) y posteriormente incorporadas en la Algoteca de la Universidad Nacional, Heredia, Costa Rica.

De acuerdo a la revisión de las muestras de campo, se reporta la presencia de la microalga Chlamydomonas vesterbottnica Skuja (Fig. 1), en todas las muestras del punto de colecta en la salida del tanque que comprende la segunda fase del tratamiento de los lixiviados.

De acuerdo a la revisión de las muestras de campo, se reporta la presencia de una especie de microalga flagelada que según sus características morfológicas se identificó como Chlamydomonas vesterbottnica Skuja (Fig.1), Ettl (1983) propone usar más este nombre como sinónimo Chloromonas vesterbottnica. Menezes \& de Mattos Bicudo

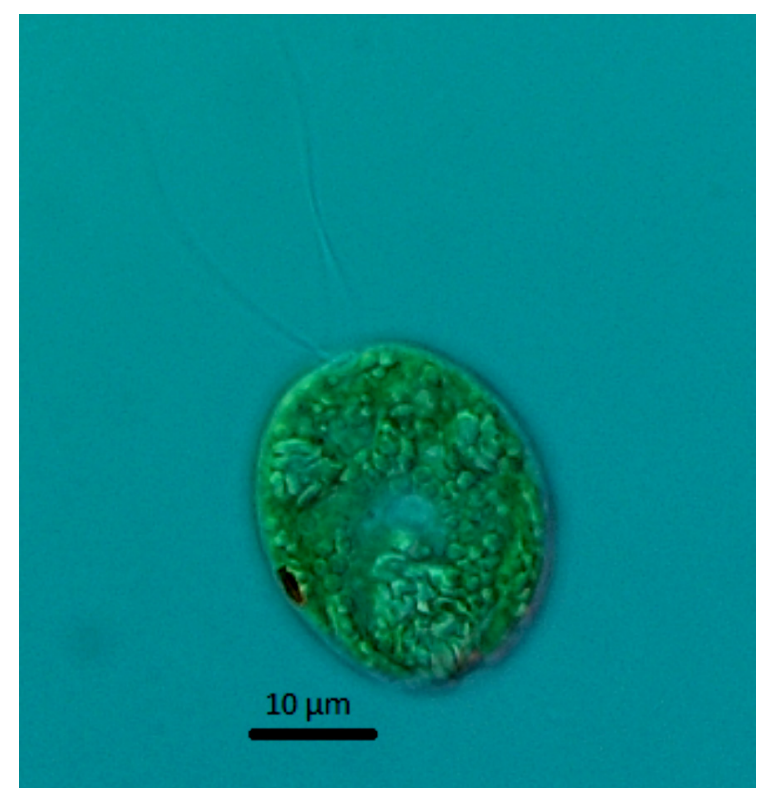

FIG. 1. Chlamydomonas vesterbottnica Skuja. 
(2008) reportan esta especie como Chloromonas cf. vesterbottnica, en su inventario florístico realizado en cuatro cuerpos de aguas en el estado de Rio de Janeiro, Brasil. Las observaciones del material en el presente estudio concuerdan con estos autores, que indican que Chamydomonas vesterbottnica Skuja es una especie que posee células de forma ancha elipsoidal a redonda elipsoidal y con paredes celulares bastante gruesas (Fig. 1). Las células presentan una papila baja de media esfera y dos flagelos que tienen la misma longitud que el cuerpo. Los cloroplastos parecen de forma de copa, sin embargo no forman una unidad, sino que se muestran en forma de rodajas, con un estigma grande en forma de raya de hasta $7 \mu \mathrm{m}$. Las células miden de 20 a $29 \mu \mathrm{m}$ de largo y de 17 a $23 \mu \mathrm{m}$ de ancho lo cual coincide con las mediciones realizadas.

Este es el primer reporte de la presencia de esta microalga en Costa Rica y su presencia toma mucha relevancia, ya que se encuentra asociada a los lixiviados generados en el relleno sanitario. Lavina, Borges de Castilhos and Ribeiro da Costa (2010) reportan encontrar microalgas del género Chlamydomonas en estanques de lagunas de estabilización, lo cual se puede relacionar con las características del estado de tratamiento que reciben dichas lagunas, en especial debido a sus requerimientos tróficos. Fernandes (2009) citado por Lavina et al. (2010), explica que la alta densidad encontrada de estos microorganismos en las lagunas se debe probablemente a que ellos se pueden adaptar con más facilidad a condiciones extremas de contaminación por lixiviados, lo cual lleva a una exclusión de otros géneros de microalgas. En un estudio realizado por Lin, Chan, Jiang and Lan (2007), también estos autores reportan Chlamydomonas asociado a los lixiviados, por lo cual despierta un especial interés para buscar posibles aplicaciones biotecnológicas que permitan encontrar soluciones viables, eficientes y de bajo costo para el tratamiento de aguas residuales problemáticas por la presencia de contaminantes.

\section{AGRADECIMIENTOS}

Especial agradecimiento para David M. John, del National History Museum of London, por su apreciable colaboración en la identificación del alga.

\section{REFERENCIAS}

Anderson, R.A. (2005). Algal culturing techniques. London, UK: Elsevier Science and Technology Books, Academic Press.

Ehrig, H.J. (1992). Cantidad y contenidos de lixiviados de rellenos de desechos domesticos. PN 88.2965.6-03.100 proyecto cepis/gtz. Coordinación Sectorial Regional para Latinoamérica en Ingeniería Sanitaria y Ambiental de la GTZ. San José, Costa Rica.

Ettl, H. (1983). Chlorophyta, I. Phytomonadina. En Süsswasserflora von Mitteleuropa, Band 9. Gustav Fischer, Stuttgart.

Giraldo, E. (2001). Tratamientos de Lixiviados de Rellenos Sanitarios: Avances Recientes. Revista de Ingeniería, Universidad de los Andes, 14, 44-55.

Graham, J.E., Wilcox, L.W. \& Graham, L.E. (2008). Algae. San Francisco, Estados Unidos: Benjamin Cummings, Pearson.

Guiry, M.D. \& Guiry, G.M. (2011). Algae Base. World-wide electronic publication, National University of Ireland, Galway. Recuperado de http://www.algaebase.org; searched on 15 of September 2011.

Harris, E. H. (2001). Chlamydomonas as a Model Organism. Annual Review Plant Physiology \& Plant Moleular Biology, $52,363-406$.

John, D.M; Whitton, B.A. \& Brook, A.J. (2002). The Freshwater Algal Floral of the British Isles. An Identification Guide to freshwater and terrestrial Algae. The Natural History Museum and The British Phycological Society. Cambridge, United Kingdom: University Press.

Lavina, M.C; Borges de Castilhos, A. \& Ribeiro da Costa, R.H. (2010). Desempenho de sistema de tratamento de lixiviado de aterro sanitário com recirculação do efluente. Performance of a sanitary landfill leachate treatment system with effluent recirculation. Revista Engenharia Sanitária, $15,401-410$

Lin, L., Chan, G.Y.S., Jiang, B.L. \& Lan, C.Y. (2007). Use of ammoniacal nitrogen tolerant microalgae in landfill leachate treatment. Waste Management, 27, 1376-1382.

Manuell, A.L. \& Mayfield, S.P. (2006). A bright future for Chlamydomonas. Genome Biology, 7(9), 327.

Menezes, M. \& de Mattos Bicudo, C.E. (2008). Flagellate green algae from four water bodies in the state of Rio de Janeiro, Southeast Brazil. Hoehnea, 35(3), 435-468.

Nozaki, H. (2003). Flagellated Green Algae. En Freshwater algae of North America. Ecology and classification, Aquatic Ecology Series. Amsterdam: Academic Press. 
